\title{
Erratum to: A two-phase algorithm for bin stretching with stretching factor 1.5
}

\author{
Martin Böhm ${ }^{1}$ • Jiří Sgall' ${ }^{1}$ - Rob van Stee ${ }^{2}$. \\ Pavel Veselý ${ }^{1}$
}

\section{Erratum to: J Comb Optim DOI 10.1007/s10878-017-0114-4}

The original title of this article is "A two-phase algorithm for bin stretching with stretching factor 1.5". The title was inadvertently changed to "A two-phase algorithm for bin stretching with stretching factor 1:5".

The original article was corrected.

The online version of the original article can be found under doi:10.1007/s10878-017-0114-4.

$凶 \quad$ Martin Böhm

bohm@iuuk.mff.cuni.cz

Jiří Sgall

sgall@iuuk.mff.cuni.cz

Rob van Stee

rob.vanstee@leicester.ac.uk

Pavel Veselý

vesely@iuuk.mff.cuni.cz

1 Computer Science Institute of Charles University, Prague, Czech Republic

2 Department of Computer Science, University of Leicester, Leicester, UK 